\title{
Ship complexity assessment at the concept design stage
}

\author{
Jean David Caprace $\cdot$ Philippe Rigo
}

Received: 18 February 2010/Accepted: 2 September 2010/Published online: 8 October 2010

(C) JASNAOE 2010

\begin{abstract}
An innovative complexity metric is introduced that provides a way to compare similar or different ship types and sizes at the contract design stage. The goal is to provide the designer with this information throughout the design process so that an efficient design is obtained during the first design run. Application to and validation on real passenger ships indicate that there is a significant correlation between the error in an engineer's judgement of complexity and the cost assessment error. It follows that this tool could be used to improve knowledge of the ship's complexity at the contract design stage, and even to try to optimise the design if the complexity criteria are not fixed by the shipowners.
\end{abstract}

Keywords Design complexity - Shipbuilding ·

Cost assessment $\cdot$ Optimisation

\section{Introduction}

\subsection{How to define complexity}

Describing and understanding complexity at the design stage remains significant problems in the shipbuilding industry. In contrast with the relative simplicity associated with a few degrees of freedom, ship behaviour cannot be gauged solely based on knowledge of the behaviour of individual ship parts.

\footnotetext{
J. D. Caprace $(\bowtie) \cdot$ P. Rigo

ANAST, University of Liège, 1 Chemin des chevreuils, 4000 Liège, Belgium

e-mail: jd.caprace@ulg.ac.be

P. Rigo

e-mail: ph.rigo@ulg.ac.be
}

Despite many years of research in this field, it is very hard to find a formal definition of a "complex system" in the literature. Complexity is a term that is normally used to describe a characteristic that is hard to define and even harder to quantify precisely.

In general usage, complexity often tends to be used to characterise something with many parts in intricate arrangements [1]. Actually, in science there are various approaches to characterising complexity, as diverse as they are different, and covering fields such as engineering, IT technology, management, economy, arithmetic, statistics, data mining, life simulation, psychology, philosophy, information, linguistics, and so on. This is just a small sample of the enormous diversity of considerations given to the concept of complexity. Many definitions tend to postulate or assume that complexity expresses a condition of numerous elements in a system and numerous forms of relationships among the elements. At the same time, what is complex and what is simple is relative and changes with time.

In a series of observations about complex systems and the architecture of complexity, [2] highlights some common characteristics:

- Most complex systems contains a lot of redundancy

- A complex system consists of many parts

- There are many relationships/interactions among the parts

- The complex system can often be described by a hierarchy; redundant components can be grouped together and considered as integrated units.

A hierarchy is a system that is composed of interrelated subsystems, each of the latter being, in turn, hierarchical in structure, until we reach the lowest level of the elementary subsystem. In their dynamics, hierarchies have 
a property-near-decomposability—-that greatly simplifies the description of a complex system, and makes it easier to understand how the information needed for the development or reproduction of the system can be stored in reasonable way.

In the everyday use of the word "complexity", a part A may be considered more complex than $\mathrm{B}$ if $\mathrm{A}$ is more difficult to design and manufacture than $\mathrm{B}$. This subjective measure of complexity is not, however, sufficient for engineering analysis.

Complexity has captured the interest of engineers for many years, and many definitions of it are given in the literature [3]. Nowadays, more and more systems and technologies contain overwhelming levels of complexity. This issue means that methods that can break them down into a more understandable form are required; hence the need to define and measure complexity.

Industry has already attempted to evaluate complexity using empirical measures. The problem is that this results in a proliferation of possible measures: typical examples include the number of items in the ship, analyses of production sequences and assemblies, etc. Having so many metrics poses problems. How do you know you are using the most appropriate ones, or that you have sufficient accuracy? How can you tell if complexity is bring reduced if one measure falls but another rises?

Various researchers have recognised the importance of objectively measuring complexity in order to help address the cause of such engineering and management-related problems [4-6]. Our first objective is to decide what complexity is; a model of how to measure it can then be produced.

\subsection{Objectives of a ship design complexity metric}

As the complexity of a ship increases, the life cycle costs (LCC) of the ship will typically increase as well. Also, a complex ship is commonly the result of a lengthy and complicated and therefore costly design process. Furthermore, because of the interconnection of various components and subassemblies in a complex ship, the engineering change process is often a complex and cumbersome task. Next, the manufacture of a complex ship entails the adaptation of complex process plans and the use of sophisticated manufacturing tools and technologies. Additionally, a complex ship requires a complex supply chain, which introduces various managerial and logistic problems. Finally, serviceability is a challenging issue for a complex ship due to the existence of numerous failure modes with multiple effects that have varying levels of predictability.

Therefore, it is beneficial to objectively measure the complexity of ships in order to systematically eliminate nonessential details. The main objective of this study is to define quantitative measures of complexity that can be evaluated for a ship model at the early stages of the project design. This measure of the complexity of a design should be able to guide the designer in creating a product with the most cost-effective balance of manufacturing and assembly difficulty. The goal is to provide the designer with such information throughout the design process, so that an efficient design is produced in the first instance.

In terms of the manufacturing processes of ships, assembly costs and the quality of the end product, complexity plays a vital role in the realisation of the best design. Unfortunately, little has been achieved in the area of complexity metrics that can be used in a useful way. One survey by Tang and Salminen [7] shows that, among a series of studies devoted to complexity, only $20 \%$ have attempted to produce some sort of quantification. Therefore, considerable further research is required to make complexity a practically useful concept for the shipbuilding industry.

One aim of this work is to develop the means to quantify the complexity of a ship and to define measures that can be used in conjunction with other metrics, such as the assessment of production cost. Another is to allow the comparison of previous ships and a possible new contract at the conceptual design stage. A cost engineer will commonly use analogy analysis to validate budgets and provide reliable bidding prices at the conceptual design stage. This technique uses a direct comparison between two similar ships and is based on the experience and knowledge of cost engineers. The effectiveness of this method depends largely upon the ability to identify differences between the intended and existing systems. The implicit assumption is that similar ships have a similar cost. By comparing ships and adjusting for differences, it is possible to achieve a valid and usable assessment. Nevertheless, this method requires tools that can identify both similarities and differences between ships. The present paper provides some resolution to this issue.

The improvement of existing cost assessment models and the comparison of previous ships with possible new contracts are focused upon in this study.

\section{Definition of ship design complexity}

With the current trend towards building more sophisticated types of vessel, a more accurate cost assessment that reflects the complexity of the ship's structure is becoming a prerequisite during the early design stages of the project.

The measurement of complexity can eliminate subjective estimations, help to detect the most cost-effective production architecture and to control the design process, and draw attention to critical changes. It is widely accepted that it is much more cost-effective to produce an initial design that is simple, feasible and easy to assemble, instead 
of rectifying the design problems after the product has reached the shop floor.

Such a model not only helps to control the design process, as it constantly draws attention to critical changes, but it also facilitates the comparison of different design alternatives. Such comparisons will ultimately allow the identification of the solution that requires the least design effort and the lowest production costs.

Several factors that influence ship macro complexity have been identified. Our research explores the complexity of different types and sizes of ships $\left(C_{\text {typ }}\right)$ as well as the complexity of ships of the same size and type but which have different arrangements and equipment $\left(C_{\text {arr }}\right)$. The complexity model is shown in Eq. 1 , where $C_{\mathrm{T}}$ represents the total complexity and $w_{1}, \ldots, w_{i}$ are numerical constants called weighting factors:

$C_{\mathrm{T}}=\frac{w_{1} C_{\mathrm{typ}}+w_{2} C_{\mathrm{arr}}}{w_{1}+w_{2}}$.

\subsection{Complexity of ships of differing types $\left(C_{\mathrm{typ}}\right)$}

A comparison of performance indicators such as complexity should take into account the sizes and types of the ships being compared and the extent to which the production is serialised and/or standardised.

The unit of measurement usually used to compare the workloads required to build ships of different sizes and types is the compensated gross tonnage (CGT). The objective of using the CGT is to adjust shipbuilding output measurements to consider work content, or differences in construction complexity for different types and sizes of ships. For example, the labour input per gross ton for passenger vessels is much higher than it is for tankers.

The CGT concept was originally proposed by shipbuilder associations and later adopted by the Organisation for Economic Co-operation and Development (OECD). The objective was to provide a more accurate measure of shipyard activity or shipbuilding workload than could be achieved by gross tonnage (GT) or deadweight ton (DWT) measurements. After the proposal of the first version in the 1980s, the CGT system underwent a number of revisions in order to improve accuracy and better reflect changes in both ship design and shipyard working methods. The version presently used was introduced in January 2007 [8]. This new system ${ }^{1}$ adopts a formula for calculating the CGT (see Eq. 2) where the coefficients $a$ and $b$ depend only on the type of ship. Using it for commercial ships over a wide range of sizes, types, and countries gives man-hour/CGT values ranging from 10 to 60 .

\footnotetext{
${ }^{1}$ Developed by the Community of European Shipyards Associations, CESA; the Shipbuilders Association of Japan, SAJ; and the Korean Shipbuilders Association, KSA.
}

$\mathrm{CGT}=a \times G T^{b}$.

Finally, the complexities of different types of ship can be measured by dividing CGT by GT. A high ratio is an indicator of more sophisticated and special ships. The higher complexity ships are passenger ships, fishing vessels and LNG carriers, while the lower complexity ships are combined carriers, oil tankers and bulk carriers.

The CGT system has significant limitations and seems to be insufficient to understand and control competitiveness [9]. Nevertheless, it has been acknowledged worldwide as the best unit of measurement of shipbuilding output devised so far. A number of studies have been published with suggestions for improving the system or extending its applicability [10, 11].

How can we assess the complexities of two ships of the same type with the same dimensions? This is a question we will try to answer in the next section. Obviously, the complexities the ships differ due to their differing internal arrangements and equipment.

\subsection{Complexity of ships of the same type $\left(C_{\mathrm{arr}}\right)$}

The complexity of ships of the same type and same size is very difficult to quantify in the early stages of the project when very little information is available. This section proposes a method to quantify the relative complexity of a ship based on a multicriteria analysis (MCA). The Preference Ranking Organization METHod for Enrichment Evaluation (PROMETHEE) has been chosen to define a complexity metric for ships [12-14].

\subsubsection{Definition of alternatives}

The outcome of any decision-making model depends on the information at its disposal, and the type of information available may vary according to the context in which the ship is operated. Therefore, it is useful for decision-making models to consider all of the information as a whole. In multiple criteria decision making (MCDM), the decisionmaking procedure is normally carried out by choosing between different elements that the decision maker must examine and assess, using a set of criteria. These elements are called alternatives. In this study, we analysed 10 different passenger ships of approximatively the same size. These ships are numbered from SHIP01 to SHIP10. In order to define an upper and a lower limit for the complexity evaluated in this study, two ideal cases have been added: the BEST ship, with the smallest complexity; and the WORST ship, with the highest complexity. All of the criteria for these two ideal cases have been respectively minimised and maximised; e.g., the value for the criterion propulsion type [2 pods, 3-4 pods, 2-4 shaft lines, mixed] 


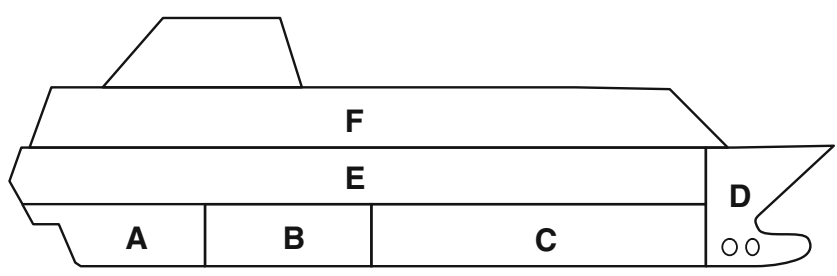

Fig. 1 Ship complexity subdivision

was chosen to be 2 pods for the BEST ship and mixed for the WORST ship.

\subsubsection{Definition of criteria}

The criteria represent the tools that enable alternatives to be compared from the point of view of complexity. It must be remembered that the selection of criteria is of prime importance in the resolution of a given problem, meaning that it is vital to identify a coherent family of criteria. The number of criteria is heavily dependent on the availability of both quantitative and qualitative information and data.

Sixty-six qualitative complexity criteria were considered in this study. These criteria were gathered into seven groups according to the location of the criterion inside the ship (see Fig. 1). A college of experts defined these criteria based on their knowledge and expertise. Only the criteria available in the early stages of the project were selected. The different groups are described below:

A The space below the watertight deck and between the engine room and the aft peak vertical bulkhead

B The engine room

C The space below the watertight deck and behind the fore peak vertical bulkhead

D The space in front of the fore peak bulkhead, including the fore strength deck

E The space above the watertight deck and below the strength deck

F The superstructures

S The whole ship.

The criteria for each group are as follows:

A Propulsion type, rudder and skeg, stern keel shape, stern keel width, hawse hole, transverse plating, framing, appendix, tank position

B Propulsion type, water intake, anti-roll keel, engine number, oil tank in DB, stiffened boxes

C Tank density, INOX tank, dynamic stabiliser, chillers

D Bulb, bow thruster, bow thruster device, washboard shape, chain hole, hawse hole, cranes

E Transom shape, large openings, overhang, tier shape, safety davit
Table 1 Definition of weights for groups of criteria in different scenarios

\begin{tabular}{lllcc}
\hline Group & \multicolumn{4}{l}{ Weight distributions for scenarios } \\
\cline { 2 - 5 } & W1 (\%) & W2 (\%) & W3 (\%) & W4 (\%) \\
\hline A & 16.7 & 14.3 & 7.6 & 5.8 \\
B & 16.7 & 14.3 & 10.2 & 7.8 \\
C & 16.7 & 14.3 & 15.5 & 11.9 \\
D & 16.7 & 14.3 & 6.9 & 5.3 \\
E & 16.7 & 14.3 & 25.3 & 19.5 \\
F & 16.7 & 14.3 & 34.5 & 26.6 \\
S & 0 & 14.3 & 0.0 & 23.0 \\
\hline
\end{tabular}

F Overhanging swimming pool, fly tower, longitudinal bulkhead, balcony, safetyboat bulkhead, safetyboat height, hull strength, aft shape, bulwark, fore slope, fore shape, number of swimming pools, openable roof, safety davit, theatre

S Weight stability balance, higher deck stress, rule class, shipowner requirement, pre-contract study, ice class, camber and sheer, fenders, load line mark, tumble home, gate location, gate lines, acoustic level, deck levels, DB height, DB level, lifts, casing long position, casing trans position, atrium.

For each criterion, the experts defined different values ordered by increasing complexity. For instance, the values for the propulsion type are 2 pods, 3-4 pods, 2-4 shaft lines, and mixed. Each qualitative criterion correspond to a number that is used to perform the MCDM.

\subsubsection{Definition of weight and scenarios}

The results of multicriteria analysis hinge on the weighting allocated and the thresholds set. The weights express the importance of each criterion and obviously can greatly influence the final outcome of the entire calculation procedure. For some authors, the problem of how to determine the weights to be assigned is still unresolved, since the various outranking methods do not specify any standard procedures or guidelines for determining them.

In this study, four scenarios with four different weight vectors were formulated to circumvent this problem (see Table 1):

1. The first scenario, $W_{1}$, representing a base case, was calculated by attributing equal weights to all criteria groups $(16.7 \%)$ without considering the criteria for group $\mathrm{S}$.

2. The second scenario, $W_{2}$, representing a base case, was calculated by attributing equal weights to all criteria groups $(14.3 \%)$. 
3. The third scenario, $W_{3}$, was calculated based on the majority opinion of the experts, but without considering the criteria for group $\mathrm{S}$.

4. The fourth scenario, $W_{4}$, was calculated based on the majority opinion of the experts. The weights used to evaluate the complexity in scenario $W_{4}$ were defined using weights of $26.6,23$ and $19.5 \%$ for groups F, S and $\mathrm{E}$, respectively.

\subsubsection{Results}

Figure 2 presents the results of multicriteria decision analysis, with the preferences (global outranking flow $\phi$ ) for various ships expressed numerically. The higher the global outranking flow the better. The small outranking flow for the SHIP04 indicates that is gives a weak performance for most criteria, whereas the high outranking flow of SHIP08 is a sign that this alternative gives mostly strong attribute values, whatever the scenario.

This result is confirmed by the spider diagram of the net flow of each criterion (see Fig. 3), which shows that SHIP08 is the strongest alternative (maximises the spider's surface) and SHIP04 is the weakest alternative (minimises the spider's surface). Changing the weights of the different criteria again yields SHIP08 as the outstanding alternative (see Fig. 2). However, this assessment technique requires human expertise and know-how. Thus, we cannot use it in all application cases.

Following the PROMETHEE method, the value of the global outranking flow $\phi$ can vary when we add new alternatives. Thus, it is important to mention here that the

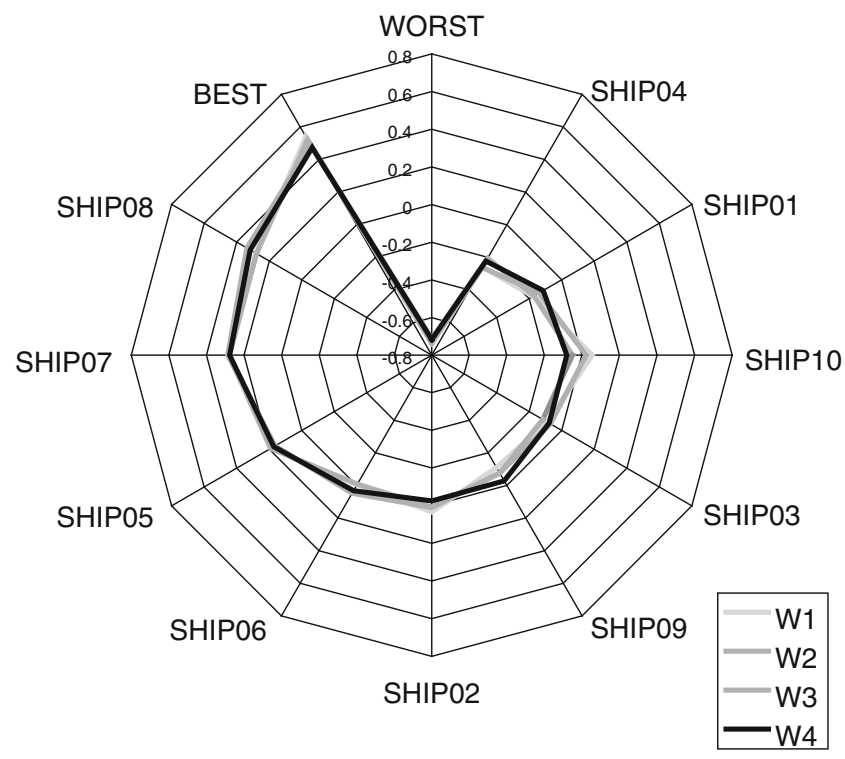

Fig. 2 Aggregated outranking flows of the alternatives value of the complexity is not an absolute value; the relative complexities of different ships are compared. Nevertheless, we have demonstrated that differences in the values of the global outranking flow $\phi$ for the same ship

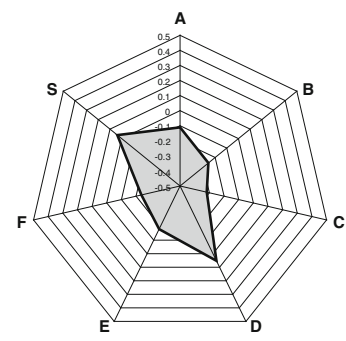

(a) SHIP01

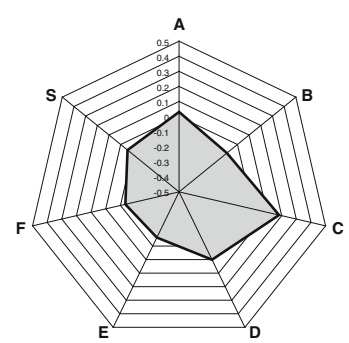

(c) SHIP03

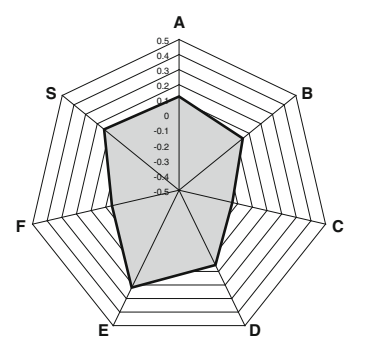

(e) SHIP05

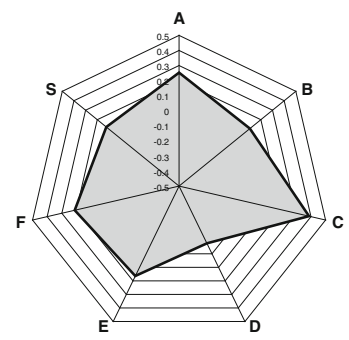

(g) $\mathrm{SHIP07}$

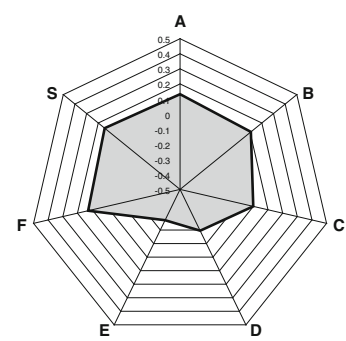

(i) SHIP09

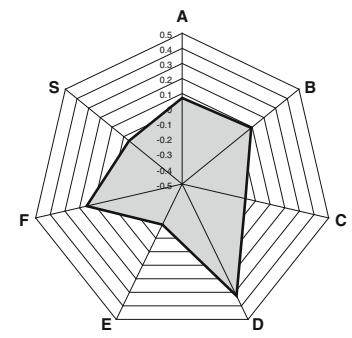

(b) SHIP02

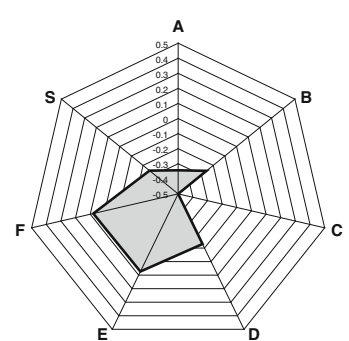

(d) SHIP04

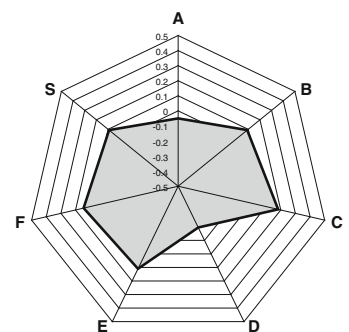

(f) SHIP06

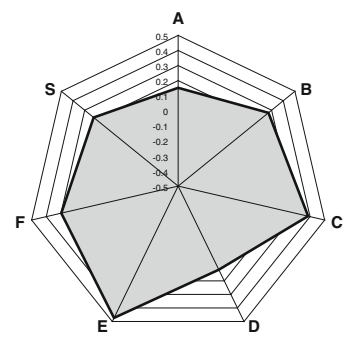

(h) SHIP08

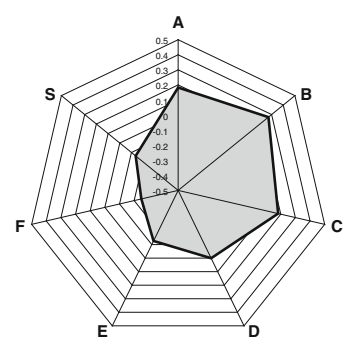

(j) SHIP10
Fig. 3 Spider representations of the ranking matrix for each alternative 
after adding a new ship to the analysis vary as $\frac{1}{(n-1)}$, where $n$ is the total number of alternatives (i.e. the total number of ships). This means that, out of 10 ships, the absolute value of the global outranking flow $\phi$ varies by $<1 \%$ when an alternative is added to the model.

Starting from the value of the global outranking flow $\phi$ for each alternative and the global outranking flows of the two ideal alternatives (i.e. the BEST ship and the WORST ship), the complexity metric $C_{\text {arr }}$ (which varies between 0 and $100 \%$ ) is defined as in Eq. 3. The results for this metric are presented in Fig. 4, where the dotted line represents expert opinion and the continuous line represents the value of the complexity metric $\left(C_{\text {arr }}\right)$.

$C_{\text {arr }}=\frac{\phi-\phi_{\mathrm{BEST}}}{\phi_{\mathrm{WORST}}-\phi_{\mathrm{BEST}}}$.

\subsubsection{GAIA visualisation}

We also carried out a GAIA visualisation, which provides a graphical representation of the various alternatives for different criteria and a $\pi$ decision axis along which the best alternative is located, which depends on the weight distribution. The GAIA plane is obtained by projecting the information in the criteria space onto a plane. The best plane is obtained by applying principal components analysis (PCA), which yields the two eigenvectors corresponding to the two largest eigenvalues of the covariance matrix of the single criterion net flows. Some information is lost when this projection is performed, but most of the information is preserved. In the present case, the preserved information amounts to $\delta=87.2 \%$.

The GAIA plane given in Fig. 5 clearly confirms the previous results. Indeed, we can observe the following characteristics:

- The criteria groups B, F and D are more discriminating than $\mathrm{E}$ and $\mathrm{C}$

- The criteria groups $\mathrm{A}, \mathrm{B}$ and $\mathrm{C}$ express similar preferences

- The criteria groups F and D express similar preferences

- The criteria groups $\mathrm{A}, \mathrm{B}$ and $\mathrm{C}$ are independent of groups F and D

- SHIP08, SHIP07 and SHIP05 are the best alternatives considering the complexity.

In order to study the behaviour of the decision model, we implemented different scenarios with different weights. For all weight distributions, the $n$ decision vector remains oriented towards the same sector of the diagram. Such weight variations can easily be handled and visualised on the GAIA plane. It is apparent that the alternatives SHIP08 and SHIP07 are still the best choices whatever the scenario.

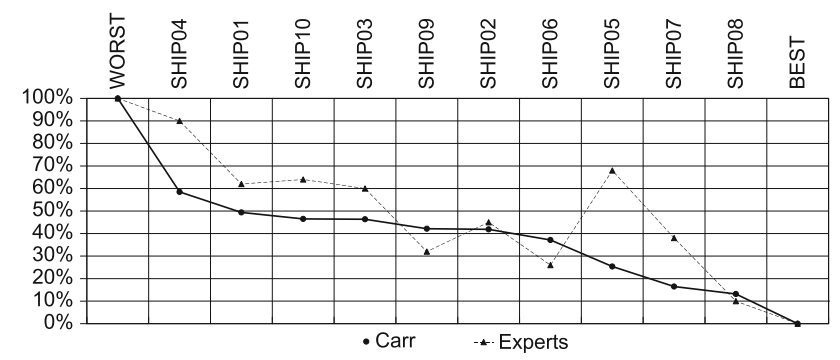

Fig. 4 Comparison of the complexity metric with expert opinion

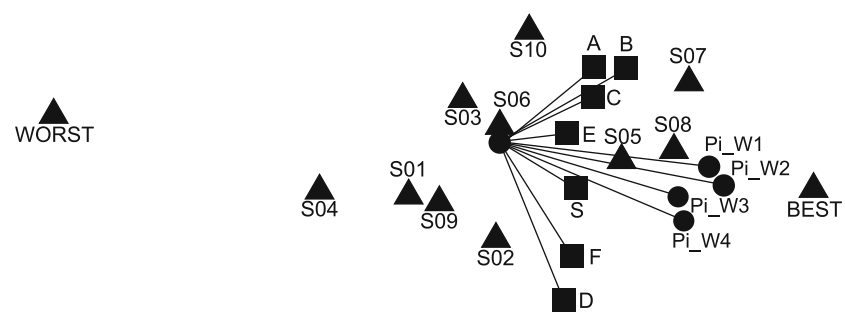

Fig. 5 GAIA view of criteria, alternatives and scenarios $(\delta=87.17 \%$, black triangles alternatives, filled circles scenarios, filled squares criteria)

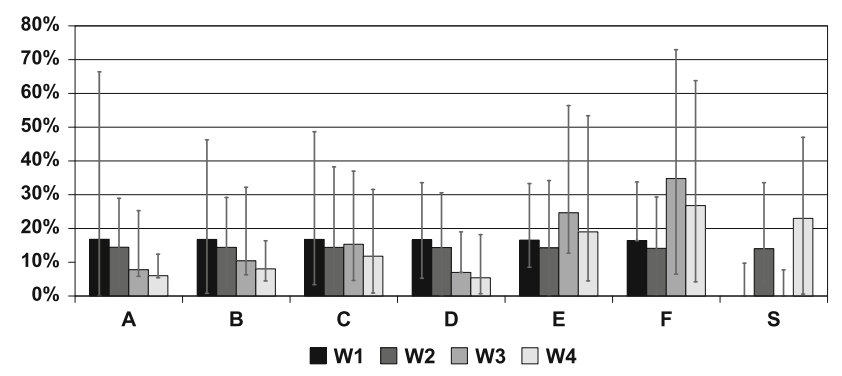

Fig. 6 Weight sensitivity analysis for each scenario

\subsubsection{Sensitivity analysis}

Sensitivity analysis has been carried out to study the subjective weights assigned to the criteria. The results presented in Fig. 6 show the high weight stability of each scenario. For each criteria group, the weight stability intervals give the limits within which the weight of the group can be modified without changing the complete $\phi$ ranking. The stability intervals are only valid when one weight is modified at a time and all of the other weights vary proportionally in order to keep the sum of the weights equal to 0 .

This result explains why the aggregated outranking flow presented in Fig. 2 does not vary much among the different scenarios.

\subsubsection{Comparison with the expert opinion}

Ten experts were questioned, and they ranked the ten studied vessels by assigning complexity values of between 


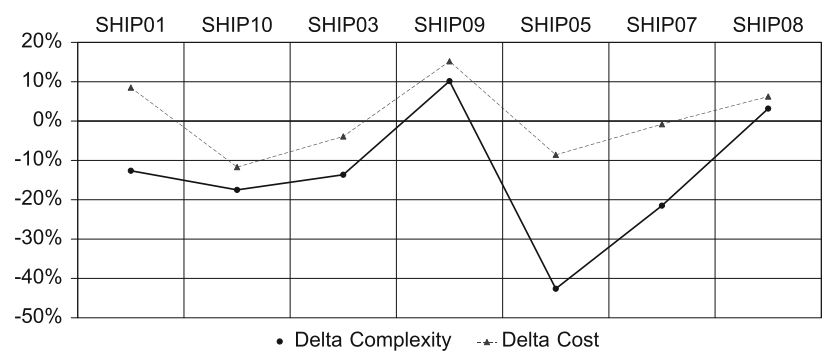

Fig. 7 Comparison between delta complexity and delta cost

0 and $100 \%$ to them based on their knowledge and experience. A comparison between the ship complexities assigned by the experts $\left(C_{\text {expert }}\right)$ and the complexities $\left(C_{\text {arr }}\right)$ assessed in this study was then carried out. Figure 4 shows the results of this comparison. We can see that the experts were relatively good at gauging the complexity of the ships, except for SHIP05. Nevertheless, the order of complexity provided by the experts is quite similar to that provided by the presented method. However, there are some differences in the quantitative values of ship complexity.

One of the aims of the study was to check whether the errors made by experts when judging vessel complexity ( $C_{\text {arr }}-C_{\text {expert }}$; also known as delta complexity) can explain the error associated with evaluating the cost of the ship at the pre-contract design stage $\left(\mathrm{Cost}_{\text {eval }}-\mathrm{Cost}_{\text {meas }}\right)$; i.e. the difference between the evaluated cost $\left(\mathrm{Cost}_{\text {eval }}\right)$ and the cost measured at the end of the project ( $\mathrm{Cost}_{\text {meas }}$ ), also known as delta cost (see Fig. 7). Note that cost errors were provided for only seven ships. There is a minor correlation $\left(R^{2}=0.601\right)$ between the error in the experts' judgement of vessel complexity and the error in the cost evaluation at the pre-contract design stage. Obviously, ship complexity cannot explain all of the differences between the evaluated and measured costs. When estimating the cost or the complexity of a ship, there is always uncertainty about the precise contents of all the items in the estimate, how the work will be performed, what the working conditions will be like when the project is executed, and so on. These uncertainties are risks associated with the project. Some refer to these risks as "known unknowns", because the estimator is aware of them and-based on past experience-can even estimate their probable values. We have shown in this study that expert misjudgement regarding ship complexity can explain at least part of the cost evaluation error.

\section{Conclusions}

Complexity can be seen as a critical problem in design that needs to be reduced as much as possible. For example, complexity is associated with difficulty in solving design problems, the combinatorial size of the search space, and the degree of variety of the generated designs. Notably, the complexity associated with solving design problems arises not only because these problems are often intractable, illdefined or ill-understood, but also because they involve many different participants, with many different goals and needs.

In order to solve these problems, different kinds of ship design complexity were investigated. Global complexity was introduced in this paper, and this provides a way to compare similar or different ship types and sizes at the contract design stage. The judgements of experts regarding ship complexity can be influenced by both ship type and size, as well as structural arrangement and type of equipment. A wrong judgement about the complexity of a ship or an inaccurate comparison of the possible new contract with a previous ship with different level of complexity during the concept design stage can lead to a dramatic reduction in profits for the shipyard. This study provides a method to exclude the engineer subjectivity by assessing the ship complexity from different aspects using PROMETHEE and sensitivity analysis. The results shown here indicate that there is a significant correlation between the error in an engineer's judgement of complexity and the cost assessment error. It follows that this tool can be used to improve knowledge of ship complexity at the contract design stage, or even to try to optimise the design if the complexity criteria are not fixed by the shipowners.

Complexity measurement is an important foundation for any systematic optimality search, which is the essential process in design. Defining and controlling the upper limit of this metric provides a good management tool for improving the overall design performance of ships.

We are well aware of the risk of creating a model that is mathematically viable but may not reflect reality due to the variety of assumptions made during the design process. The idea, nevertheless, is to define a model that can make complexity more approachable, and perhaps even practical. Nobody has ever succeeded in giving a definition of complexity that is meaningful enough to enable the complexity of a system to be measured. Ships cannot and should not be reduced to one single complexity measure. A ship is not simply an end result; it is also an entire system of manufacturing, transport and economic evolution. Complexity should be seen as a tool that aids decision making.

\section{Future work}

Previous research studies have been limited to:

- Large passenger ships

- Some ship systems (hull structure, power system, general arrangement, etc.)

- The final product without the supply chain and logistics. 
These limitations may prevent extensive use of the methodology. Additional research and development are thus required to overcome these limitations.

The present research proposes to extend the previous developments in the following ways. Improving the methodology to fully include outfitting components such as HVAC design, piping design and electrical design is vital. At the moment, European shipyards mainly produce ships with high added value because the less complex vessels are usually produced in Asia, where labour is cheaper. In this type of ship, equipment (cables, plumbing, ventilation pipes, siding, furniture, etc.) and the work involved in setting up this equipment represent a significant portion of the total price of the vessel. For example, $80 \%$ of the price of a cruise ship is related to its equipment. It therefore appears to be necessary to include these elements in the developed tools.

It should also be recognised that the complexity involved in manufacturing ships and offshore structures is closely related to problems with the delivery of equipment or facilities and the ability to predict early supply from vendors. Furthermore, the management of subcontractors for design and production also contributes to the overall complexity. Assessments of these types of difficulties should also be incorporated into the previous developments.

Acknowledgments The authors thank the University of Liege and experts at some European shipyards for their collaboration with this project, as well as the Belgian National Funds of Scientific Research (NFSR) for their financial support.

\section{References}

1. Simon HA (1962) The architecture of complexity. Proc Am Philos Soc 106:467-482
2. Simon H (1996) The sciences of the artificial. MIT Press, Cambridge

3. Rodriguez-Toro C, Tate S, Jared G, Swift K (2003) Complexity metrics for design. Proc Inst Mech Eng B 217:721-725

4. Chryssolouris G (1994) Measuring complexity in manufacturing systems (technical report). University of Patras, Patras

5. Little G, Tuttle D, Clark DER, Corney J (1997) A feature complexity index. Proc Inst Mech Eng C 212:405-412

6. Calinescu A, Efstathiou J, Sivadasan S, Schirn J, Huaccho HL (2000) Complexity in manufacturing: an information theoretic approach. In: Conference on complexity and complex systems in industry. University of Warwick, Warwick, 19-20 Sept 2000, pp 19-20

7. Tang V, Salminen V (2001) Towards a theory of complicatedness: framework for complex systems analysis and design. In: 13th International conference on engineering design, Glasgow, UK, 21-23 August 2001, p 8

8. Community of European Shipyards Associations (CESA), the Shipbuilders' Association of Japan (SAJ) and the Korean Shipbuilders Association (KSA) (2007) Compensated gross ton (CGT) system (technical report). Organisation for Economic Co-Operation and Development (OECD), Paris. http://www.oecd. org/dataoecd/59/49/37655301.pdf

9. Bertram V (2003) Strategic control of productivity and other competitiveness parameters. Proc Inst Mech Eng M 217:61-70

10. Bruce GJA (2006) A review of the use of compensated gross tonnage for shipbuilding performance measurement. J Ship Product 22:99-104

11. Lamb T (2003) Methodology used to calculate naval compensated gross tonnage factors. J Ship Product 19:29-30

12. Brans J, Mareschal B (1992) PROMETHEE V: MCDM problems with segmentation constraints. INFOR 30:85-96

13. Brans J, Mareschal B (1994) How to decide with PROMETHEE. http://www.visualdecision.com/Pdf/How\%20to\%20use $\% 20$ PROMETHEE.pdf

14. Brans J-P, Mareschal B (2005) PROMOTHEE methods. In: Figueira J, Greco S, Ehrgott M (eds) Multiple criteria decision analysis: state of the art surveys. Springer, New York, 78:163-186 